\title{
Use of a Fibrin Sealant Patch at Cesarean for Conservative Management of Morbidly Adherent Placenta
}

\author{
James A. Greenberg, MD ${ }^{1}$ Julian N. Robinson, MD $^{1}$ Jean M. Carabuena, MD ${ }^{2}$ Michaela K. Farber, MD ${ }^{2}$ \\ Daniela A. Carusi, MD ${ }^{1}$ \\ ${ }^{1}$ Department of Obstetrics and Gynecology, Brigham and Women's \\ Hospital, Boston, Massachusetts \\ 2 Department of Anesthesia, Brigham and Women's Hospital, Harvard \\ Medical School, Boston, Massachusetts \\ Address for correspondence James A. Greenberg, MD, Department of \\ Obstetrics and Gynecology, Brigham and Women's Hospital, 1153 \\ Centre Street, Suite 3C, Boston, MA 02130 \\ (e-mail: jagreenberg@bwh.harvard.edu).
}

Am J Perinatol Rep 2018;8:e325-e327.

\begin{abstract}
Keywords

- fibrin patch

- placenta accrete

- morbidly adherent placenta

- postpartum hemorrhage

- cesarean

Background Morbidly adherent placenta represents a surgical challenge and source of maternal morbidity and mortality. We report the use of a fibrin sealant patch to address hemorrhage associated with a morbidly adherent placenta during cesarean delivery.

Case A patient underwent repeat cesarean delivery with complete anterior placenta previa and anticipated morbidly adherent placenta. Bleeding persisted following delivery and removal of the placenta, despite uterine artery embolization. A fibrin sealant patch was applied as an adjuvant intervention to the placental bed and hemostasis was achieved without resorting to a hysterectomy.

Conclusion Postpartum hemorrhage is an ongoing leading source of maternal morbidity and mortality. A case is presented in which a fibrin sealant patch provided control of focal placental bed bleeding, allowing removal of a focal morbidly adherent placenta and avoidance of hysterectomy.
\end{abstract}

Morbidly adherent placenta represents a profound surgical challenge and source of maternal morbidity and mortality associated with postpartum hemorrhage. In a cohort study including 25 hospitals, 158 patients with morbidly adherent placenta were identified among 115,502 women (1 per 731 births) with a median estimated blood loss (EBL) of 2,000 $\mathrm{mL}, \mathrm{ICU}$ (intensive care unit) admission rate of 31 , and $70 \%$ hysterectomy rate. ${ }^{1}$ While hysterectomy is the mainstay of morbidly adherent placenta management, a growing body of literature has described uterine conservation. While leaving the adherent placenta in situ leads to less immediate blood loss than placental removal, it carries delayed risks of hemorrhage and infection. ${ }^{2}$ Reported techniques to successfully remove the adherent placenta at delivery generally involve complex surgical techniques. ${ }^{3}$ Simpler measures to achieve hemostasis during cesarean delivery after removal of an adherent placenta are needed.

received

May 31, 2018

accepted after revision

October 6, 2018
In 2012, the FDA (Food and Drug Administration) approved the Evarrest Fibrin Sealant Patch (Ethicon, Inc Somerville, NJ) as an adjunct to hemostasis for soft tissue bleeding. The patch consists of human fibrinogen and human thrombin embedded on oxidized regenerated cellulose in a woven polyglactin-910 (Vicryl) patch. ${ }^{4}$ While this patch has not been described in obstetric surgery, several reports have demonstrated successful off-label use of a similar fibrin patch in a variety of obstetric hemorrhage situations. ${ }^{5-7}$ We now report the predelivery plan and use of a fibrin sealant patch to facilitate hemostasis in a case of confirmed morbidly adherent placenta.

\section{Case Report}

A 35-year-old gravida 5, para 2 presented at 36 weeks of gestation for a repeat cesarean delivery with a complete

Copyright $\odot 2018$ by Thieme Medical Publishers, Inc., 333 Seventh Avenue, New York, NY 10001, USA. Tel: +1(212) 584-4662.
License terms

(1) (1) $\Theta \circledast$
DOI https://doi.org/

10.1055/s-0038-1675848. ISSN 2157-6998. 
anterior placenta previa. Antepartum ultrasound suggested a focal placenta accreta with a hypervascular lower uterine segment (-Fig. 1). Her one prior cesarean delivery was complicated by an unanticipated morbidly adherent placenta that was manually removed with an EBL of 1,500 mL. Despite receiving counseling about the risks of morbidly adherent placenta, hemorrhage, and hysterectomy, the patient repeatedly expressed a strong desire for repeat uterine conservation. She preferred placental removal instead of placental retention for management and she consented to the use of intraoperative uterine artery embolization with the use of a fibrin sealant patch as a final option to avoid hysterectomy if standard surgical interventions were unsuccessful. Her preoperative hemoglobin was $11.2 \mathrm{~g} / \mathrm{dL}$.

Following induction of epidural anesthesia and prior to cesarean delivery, bilateral ureteral stents were placed cystoscopically and a right femoral artery sheath was placed by the interventional radiologist. Cesarean delivery was performed through midline vertical skin and vertical uterine incisions. The placenta was noted to be adherent to the anterior myometrium beneath this incision but could be partially displaced to allow delivery of a healthy infant. At this point there was no active hemorrhage and the hysterotomy was closed in a single layer with the placenta left in situ.

The surgical field was covered with sterile drapes and selective bilateral uterine artery embolization was performed to stasis, as confirmed by fluoroscopy. The obstetric team then reopened the hysterotomy and forcibly removed the placenta. No separation plane could be created in the anterior lower uterine segment. As much of the placenta that could be removed was removed from this area in multiple fragments. Subsequently, a partial-thickness defect was observed with brisk bleeding from this area of the uterus. A $2 \times 2$ inch piece of fibrin sealant patch was placed over the area of bleeding and held firmly in place with a moist sponge for 3 minutes. Upon reinspection, the bleeding was wellcontrolled (-Fig. 2). The uterus was closed with the fibrin

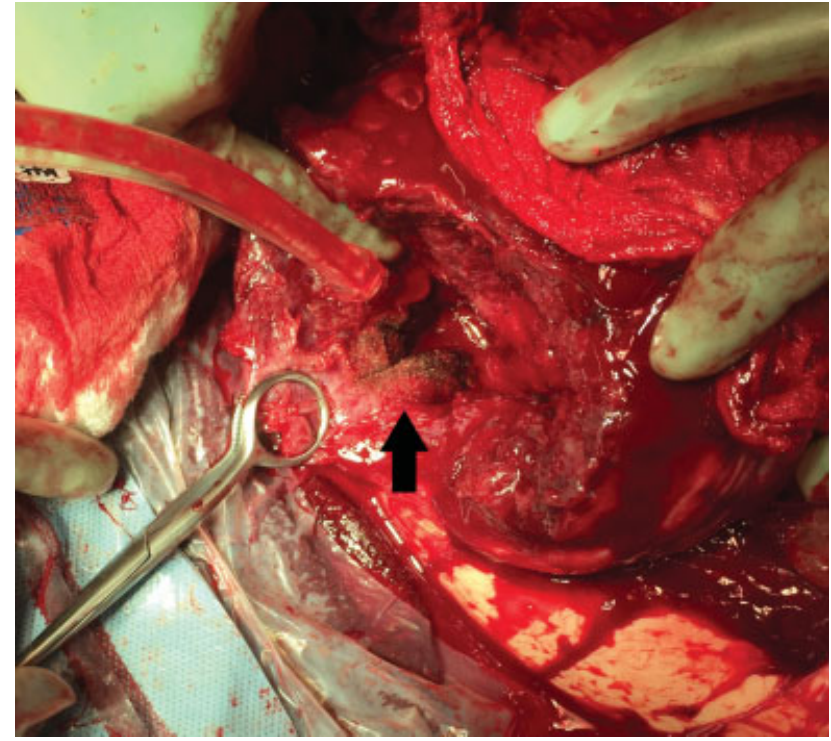

Fig. 2 Hysterotomy with fibrin sealant patch (arrow) on the anterior uterine wall over the site of bleeding.

patch left in situ and the remainder of the case was routine. The EBL was $1,800 \mathrm{~mL}$. The patient was hemodynamically stable, required no intraoperative transfusion of blood products, and was transferred to the labor and delivery unit for recovery. She received 1 unit of packed red blood cells for symptomatic anemia and a hemoglobin of $7.7 \mathrm{~g} / \mathrm{dL}$ on postoperative day 3 . She was otherwise discharged home routinely on postoperative day 4 . On postoperative day 13 , she noted an asthma exacerbation and developed urticaria and pruritis that responded to oral antihistamine therapy. At her routine visit 6 weeks postpartum, she was doing well with no further bleeding and a hemoglobin of $13.4 \mathrm{~g} / \mathrm{dL}$. An ultrasound showed a heterogenous thickened anterior myometrium but no evidence of retained products of conception.

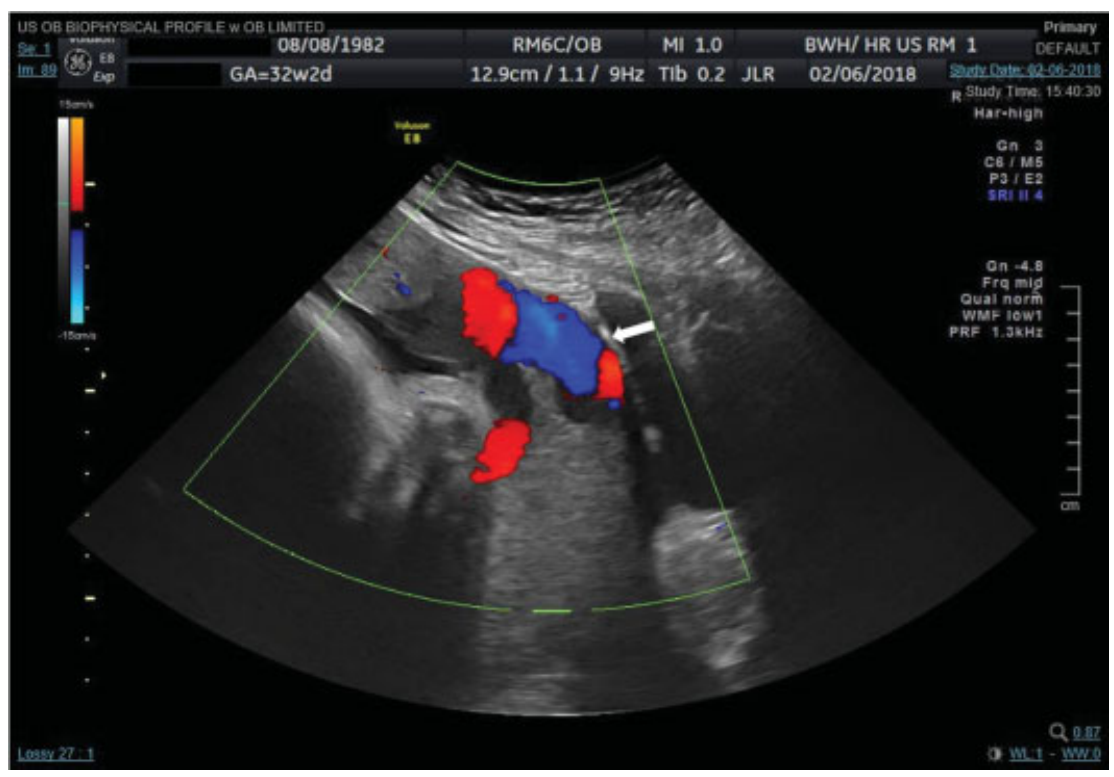

Fig. 1 Anterior placenta with large venous lakes (arrow) in the lower uterine segment. 


\section{Discussion}

The introduction of a fibrin-based, manually applied patch to obstetricians' armamentarium for obstetric surgical bleeding offers a promising potential development, with established efficacy for soft tissue bleeding and relative ease of use. Here we have demonstrated a case in which the patch, in addition to uterine artery embolization and pressure, provided control of focal placental bed bleeding, allowing removal of a focal morbidly adherent placenta and avoidance of hysterectomy.

A fibrin sealant patch (TachoSil Fibrin Sealant Patch, Baxter Healthcare Corp, Westlake Village, CA) was first approved in 2010 for use in cardiovascular and hepatic surgery. ${ }^{8}$ This patch, which embeds human fibrin and thrombin in an equine collagen bed, has been described for off-label obstetric use with both external uterine and placental bed bleeding. ${ }^{5,7}$ We identified one report of its use during cesarean delivery for morbidly adherent placenta in which EBL was $4 \mathrm{~L}$ but uterine conservation was achieved. Our patient had a more modest hemorrhage with an $1,800 \mathrm{~mL}$ EBL and 1 unit packed red blood cell transfusion which may reflect the preceding use of uterine artery embolization and preplanned immediate application of the topical hemostatic patch. Her only postoperative medical issue was urticaria which was managed uneventfully with oral antihistamines. Hypersensitivity has been a described side-effect of fibrin sealant patch use and cannot be excluded in this case. ${ }^{4,8}$ Given that fibrin sealant patches can cause hypersensitivity reactions, caution should be used before choosing this therapy for atopic patients at risk for hypersensitivity reactions. Further, providers should be aware that because the biological components of the fibrin sealant patch are made from human plasma, it may carry a risk of transmitting infectious agents, for example, viruses, the variant Creutzfeldt-Jakob disease (vCJD) agent and theoretically, the Creutzfeldt-Jakob disease (CJD) agent. ${ }^{4}$

Only a minority of patients with morbidly adherent placenta are managed successfully with uterine conservation, though many may desire retention of the uterus for future fertility, adherence to cultural norms, and reduction of surgery-related morbidity. Studies have shown more hemorrhage with attempts to remove the placenta, ${ }^{2}$ leading some to recommend that the adherent placenta never be forcibly removed. ${ }^{3}$ Alternatively, the risks of delayed hemorrhage and infection with placental retention may be undesirable for many patients. Surgical techniques for focal placental excision and uterine reconstruction have been described but are either unfamiliar and/or untenable to most surgeons. ${ }^{3}$ The use of uterine artery embolization prior to attempted adherent placental removal has not been well studied in a controlled fashion and was insufficient to control our patient's postpartum hemorrhage. The fibrin sealant patch offers a promising, simple to use, addition to conservative measures.
While our patient's morbidly adherent placenta was suspected in the antepartum period, ultrasound often misses focal areas of placental adherence or invasion, and surgical teams may not be prepared for more sophisticated surgical and interventional radiology techniques to control bleeding. Thus, the simplicity of this method may bring a conservative management option to more patients who are diagnosed intraoperatively with this condition or any other focal bleeding that is encountered at cesarean delivery. We combined the patch with bilateral uterine artery embolization to offer this patient the standard conservative management techniques currently used at our center. Since a combination of methods was used in this case, we cannot be sure that the fibrin patch was singularly responsible for the cessation of bleeding. However, because placental bleeding is typically diffuse but low-pressure, fibrin patches may be efficacious without embolization in some cases. ${ }^{5}$ Further research is warranted to demonstrate the success and safety of this technique for obstetric bleeding and to further establish the long-term effects on the uterus.

\section{Conflict of Interest}

None.

\section{References}

1 Bailit JL, Grobman WA, Rice MM, et al; Eunice Kennedy Shriver National Institute of Child Health and Human Development (NICHD) Maternal-Fetal Medicine Units (MFMU) Network Morbidly adherent placenta treatments and outcomes. Obstet Gynecol 2015;125(03):683-689

2 Perez-Delboy A, Wright JD. Surgical management of placenta accreta: to leave or remove the placenta? BJOG 2014;121(02): 163-169, discussion 169-170

3 Sentilhes L, Kayem G, Chandraharan E, Palacios-Jaraquemada J, Jauniaux E; FIGO Placenta Accreta Diagnosis and Management Expert Consensus Panel FIGO consensus guidelines on placenta accreta spectrum disorders: conservative management. Int J Gynaecol Obstet 2018;140(03):291-298

4 Evarrest Fibrin Sealant Patch: prescribing information. 2017. Available from: https://hostedvl106.quosavl.com/qb/doc/ n9kjvl0hvn14pei0kgrktcibp8. Accessed April 21, 2018

5 Fuglsang K, Petersen LK. New local hemostatic treatment for postpartum hemorrhage caused by placenta previa at cesarean section. Acta Obstet Gynecol Scand 2010;89(10):1346-1349

6 Shirata I, Fujiwaki R, Takubo K, Shibukawa T, Sawada K. Successful continuation of pregnancy after repair of a midgestational uterine rupture with the use of a fibrin-coated collagen fleece (TachoComb) in a primigravid woman with no known risk factors. Am J Obstet Gynecol 2007;197(04):e7-e9

7 Tinelli A. Post-cesarean section hemorrhage treated by a collagen patch coated with the human coagulation factors. J Clin Case Rep 2011;1(01):1-4

8 TachoSil Fibrin Sealant Patch: full prescribing information. 2015. Available from: https://www.fda.gov/downloads/BiologicsBloodVaccines/BloodBloodProducts/ApprovedProducts/LicensedProductsBLAs/FractionatedPlasmaProducts/UCM207483.pdf. Accessed April 21, 2018 